\title{
Magnetic Resonance Imaging of the Pelvis without Contrast
}

National Cancer Institute

\section{Source}

National Cancer Institute. Magnetic Resonance Imaging of the Pelvis without Contrast. NCI Thesaurus. Code C137870.

Magnetic resonance imaging of the pelvis without the use of a contrast agent. 